\title{
Common Hepatic Artery Ligation for Penetrating Injury
}

\author{
Dong Hun Kim¹, Jeongseok Yun ${ }^{1}$, Jae-Wook Ryu² \\ 'Department of Surgery, '2Department of Thoracic and Cardiovascular Surgery, Trauma Center, Dankook University Hospital, \\ Cheonan, Korea
}

Ligation for the injured major artery of the abdomen can be a safe and simple damage control surgical technique. We report successful surgical management of the common hepatic artery ( $\mathrm{CHA})$ transection by ligation in a 56-year-old man. The patient presented with hypotension due to a gunshot wound of the epigastrium. After preoperative imaging and intraoperative manipulation confirmed that intrahepatic arterial flow was preserved through collateral pathways, ligation of the $\mathrm{CHA}$ was performed.

(Trauma Image Proced 2016(1):18-20)

Key Words: Hepatic artery; Wounds, Penetrating; Ligation; Collateral Circulation

\section{CASE}

A 56-year-old man attempted suicide with an air gun and sustained a penetrating abdominal injury. On arrival, he was alert with hypotension. The injury severity score was 29. Physical examination of the abdomen revealed a gunshot wound entrance in the epigastric area (Fig. 1.). Abdominal computed tomography showed liver laceration in the left lateral segment, fluid collection in the perihepatic and perisplenic spaces, and central retroperitoneal hematoma in the retropancreatic area with contrast extravasation of the common hepatic artery (CHA) (Fig. 2.). Intraoperatively, a ballistic trajectory penetrated the diaphragm, segment III of the liver, and the CHA in the superior margin of the pancreatic head and reached the first vertebral body of the lumbar spine (Fig. 3.). Ligation for the CHA transection was performed with Prolene 4-0 suture and clipping while temporary control was achieved with digital compression (Fig. 3B). Backflow to the liver from the gastroduodenal artery was confirmed by palpation before and after ligation of the CHA. The patient made a full recovery without any complications.

\section{DISCUSSION}

Temporary vascular shunting can replace immediate definitive repair as a damage control procedure in vascular trauma. However, if preservation of collateral arterial flow is ascertained in the abdominal vessel, ligation of the arterial injury can be a simpler and quicker damage control procedure. The main goal in the treatment of a CHA injury is the preservation of arterial flow to the liver. Ligation of the CHA should be considered if intrahepatic arterial flow is preserved through collateral pathways (1). After the CHA ligation, in theory,

Received: May 18, 2016 Revised: June 17, 2016 Accepted: July 5, 2016

Correspondence to: Dong Hun Kim, Department of Surgery, Trauma Center, Dankook University Hospital, 201 Manghyang-ro, Dongnam-gu, Cheonan, Chungnam 330-715, Korea

Tel: 82-41-550-7119, Fax: 82-41-550-0039, E-mail: saint7331@gmail.com

Copyright (c) 2016 Korean Association for Research, Procedures and Education on Trauma. All rights reserved.

(C) This is an open-access article distributed under the terms of the Creative Commons Attribution Non-Commercial License (http://creativecommons.org/ licenses/by-nc/4.0) which permits unrestricted noncommercial use, distribution, and reproduction in any medium, provided the original work is properly cited 


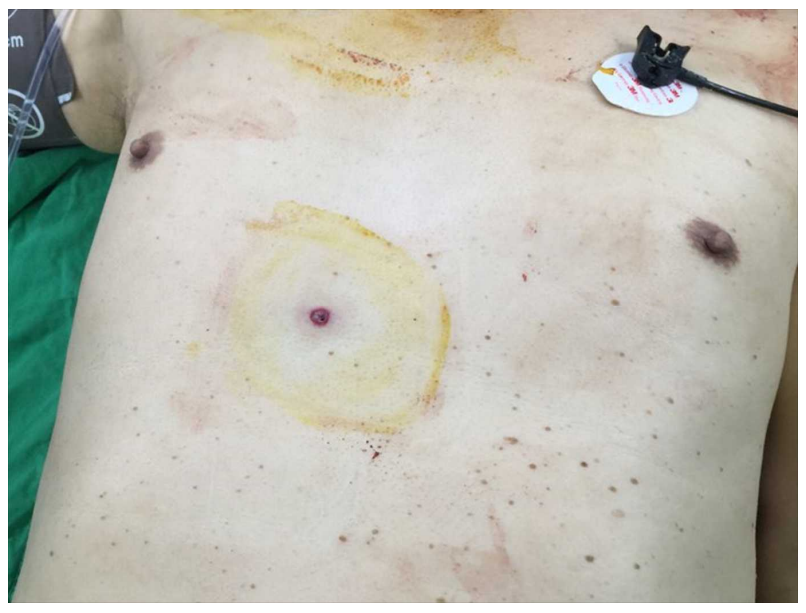

Fig. 1. Entrance wound of the gunshot at the epigastrium.

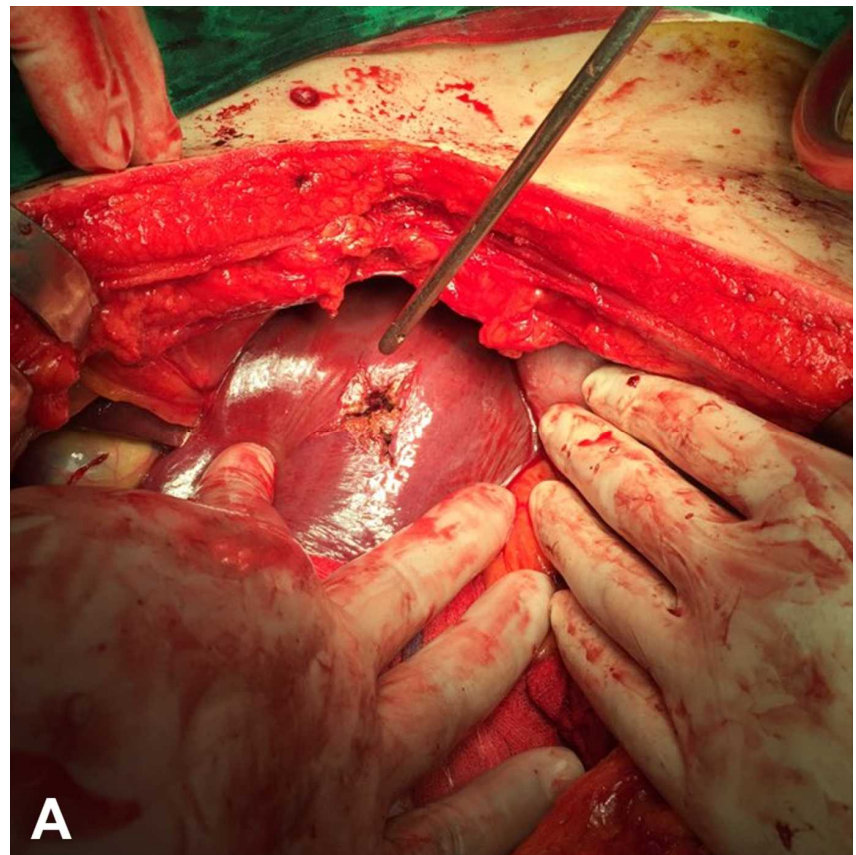

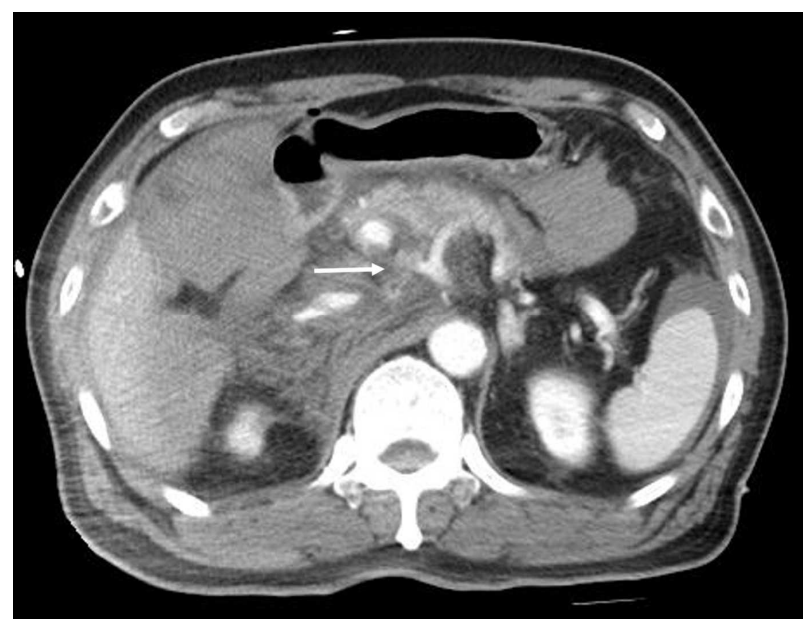

Fig. 2. Abdominal computed tomography shows ongoing bleeding of the common hepatic artery with extravasation (white arrow) and central retroperitoneal hematoma.

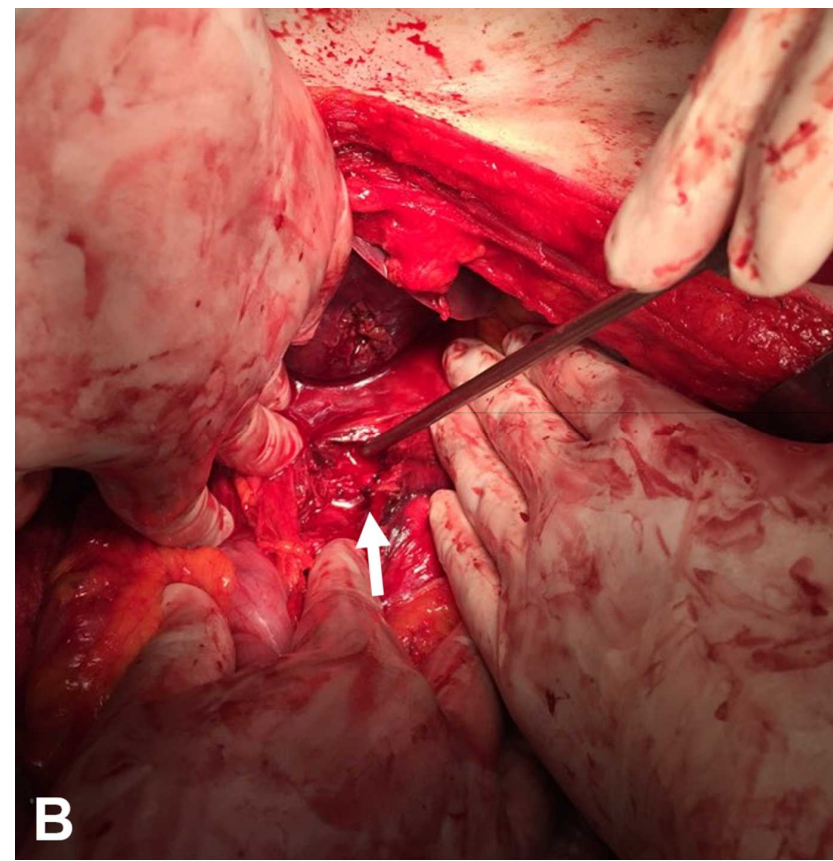

Fig. 3. Operative findings (A) A penetrating wound on the left lateral segment of the liver (B) ligation of the common hepatic artery with suture and clipping (white arrow) through a window exposed by dissection of the lesser omentum

different collateral pathways may replace arterial flow to the liver (2): [1] backflow from the superior mesenteric artery by way of the inferior pancreaticoduodenal arcades and the gastroduodenal artery, [2] arterial flow from the left gastric artery by way of the arterial circle of the lesser curvature and the right gastric artery, [3] the pericholedocal arteries; and [4] both round and triangular falciform ligaments. These collaterals to the liver should be evaluated on preoperative imaging and through intraoperative manipulations such as arterial palpation or Doppler ultrasonography. 


\section{CONFLICT OF INTEREST}

No potential conflict of interest relevant to this article was reported.

\section{REFERENCES}

1. Chirica M, Alkofer B, Sauvanet A, Vullierme MP, Levy Y,
Belghiti J. Hepatic artery ligation: a simple and safe technique to treat extrahepatic aneurysms of the hepatic artery. American journal of surgery. 2008;196(3):333-8.

2. Majno PE, Pretre R, Mentha G, Morel P. Operative injury to the hepatic artery. Consequences of a biliary-enteric anastomosis and principles for rational management. Archives of surgery. 1996;131(2):211-5. 